\title{
Die Abhängigkeit des Jo. Antiochenus von Jo. Malalas.
}

Bentley hat in der Epistola ad Millium p. 7/11 (Malal. Ed. Bonn.

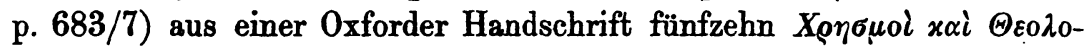

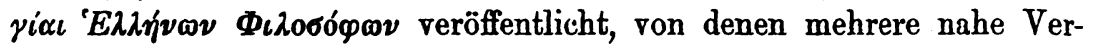
wandtschaft mit Orakeln zeigen, die von Malalas überliefert worden sind. Bentley hat sich bei diesen letzteren mit der Angabe der Überschriften und der Anfangsworte begnügt und für die Fortsetzung auf Malalàs verwiesen. Diese Stücke stammen aber möglicherweise nur mittelbar-aus Malalas, denn das Orakel, welches der ägyptische König Thulis erhalten haben soll (No 15), stammt aus Jo. Antiochenus.

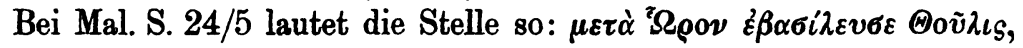

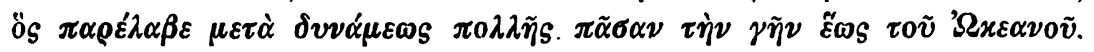

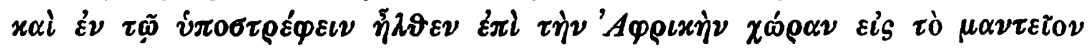

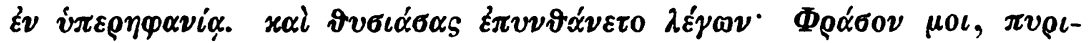
$\sigma \vartheta \varepsilon \nu \varepsilon_{\mathcal{G}}, x \tau \lambda$. Diese Fassung ist gesichert durch das Chron. Pasch. S. 83, der Malalastext des Cod. Paris. 1336 bei Cramer Anecd. Paris. II S. 237 ist gekürzt; von Malalas abhängig sind auch Kedr. S. 36 und Fr. 6, 9 des Codex Paris. 1630, dessen Exzerpte Müller sämtlich unter die des Jo. Antiochenus aufgenommen hat. Ich habe aber in meinem Programm 1892 S. 3 nachgewiesen, dafs die Exzerpte des Paris. 1630 erst von Fr. 6, 14 ab aus Jo. Antiochenus genommen sind, dafs bis dahin der Exzerptor zwar auch einen Jo. Antiochenus, hauptsächlich aber einen Malalas benutzt hat. Bei dieser Gelegenheit bemerke ich, dafs Müller den Paris. 1630 nicht als erster benutzt hat; in den Notae in Chron. Pasch. (II p. 249 ff.) ist ein Codex Regius verwertet worden, der, wie der Wortlaut der zitierten Stellen beweist, von dem Paris. 1630 nicht verschieden gewesen ist.

Mit keinem der erwähnten Texte stimmt das von Bentley veröffentlichte Orakel, wohl aber zeigt es dieselbe Fassung, wie die Suidasglosse Qoṽdıs. Bentley selbst hat auf diese Glosse verwiesen und in ihr

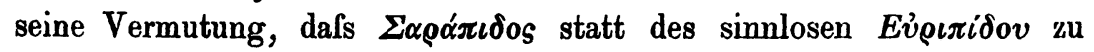
lesen sei, bestätigt gefunden. 


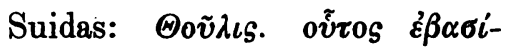

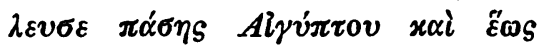

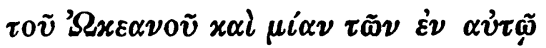

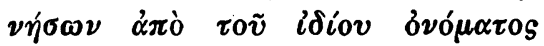

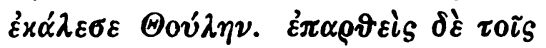

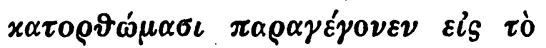

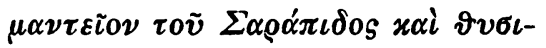

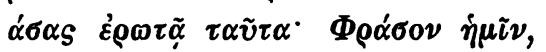
$\pi v \varrho\llcorner\sigma \vartheta \varepsilon \nu \varepsilon ́ s, x \tau \lambda$.

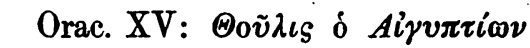
$\beta \alpha \sigma \iota \lambda \varepsilon \dot{v} s$

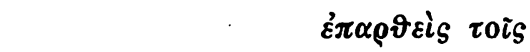

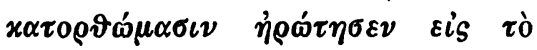

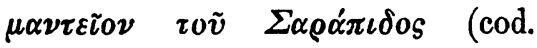

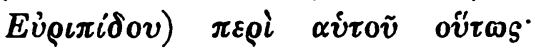
$\Phi \varrho \alpha ́ \sigma 0 \nu \mu 0 \iota, \pi v \varrho \iota \sigma \vartheta \varepsilon v \varepsilon ́ s$, etc. ut Malalas.

Beide Texte haben dieselbe von Malalas abweichende Fassung und nennen das Orakel des Serapis, beide Texte stammen also aus demselben Autor. Dieser ist aber Jo. Antiochenus. Schon Gelzer hat I S. 81/2 diese Suidasglosse im Verein mit mehreren anderen, die er nach den salmasischen Exzerpten bestimmte, für Jo. Antiochenus in Anspruch genommen.

Wenn aber dieses Orakel aus Jo. Antiochenus genommen ist, dann sind es vielleicht auch die übrigen, und diese Möglichkeit lärst die vollständige Veröffentlichung dieser Orakel als wünschenswert erscheinen. Ich will deshalb für denjenigen, der sich dieser geringen Mühe unterziehen will, das Material zusammenstellen. Die von Bentley benutzte Handschrift ist der Cod. Baroccianus 50, membran., saec. forsan XI; sie zählt 16 Nummern auf, aber No 10 und 11 gehören zusammen.

No II u. III: Vgl. Mal. S. $26 / 7=$ Chron. Pasch. S. $85 / 6=$ Paris. 1336 Cramer S. 238; ferner Kedr. S. 36/7 und Fr. 6, 11 u. 12 aus Paris. 1630. - Die Fassung des Jo. Antiochenus ist zu erschliefsen aus Fr. Salmas. bei Cramer Anecd. Paris Il S. 387, 9/21 und Suidas v. ${ }^{~ E \varrho \mu \eta ̃ ̃ s ~}$

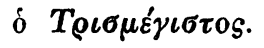

No V scheint vollständig abyedruckt zu sein. Es geht unter der

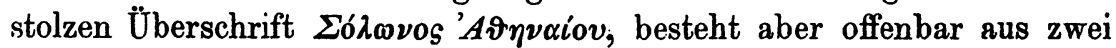
getrennten Malalasstücken. Die Einführungsworte stimmen mit Mal. S. $26,18 / 9=$ Chr. P. S. $85,16 / 7=$ Cramer S. $238,8 / 10$, fehlen aber,

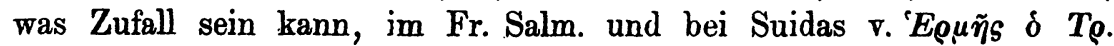
Das eigentliche Orakel stimmt mit Mal. S. $65 / 6=$ Cramer S. 241, 18/26, ferner mit Kedr. S. 73/4.

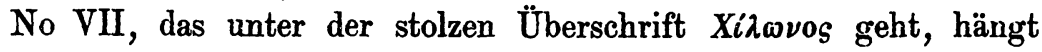
auch -mit Mal. S. 27, 1/7 = Chr. P. S. $85 / 6=$ Cramer S. 238, 12/7 irgendwie zusammen, vgl. auch Fr. Salm. S. 387, 11/6. - Die Überschriften der letzten beiden Orakel und ihre Herkunft aus Malalas zeigen deutlich, dafs dieser Spruchsammler ein frommer Fälscher war.

No X: Vgl. Mal. S. 77, 15 und Kedr. S. 209, 18. Die Fassung des 
Jo. Antiochenus liegt wahrscheinlich im Fr. 15, 1 aus Paris. 1630 vor, jedoch fehlt hier, was durch Kürzung des Exzerptors geschehen sein kann, die direkte Anrede. - In der Handschrift folgt unter No $\iota \alpha^{\prime}$ die Fortsetzung.

$\mathrm{Ob}$ in diesen Sprüchen allen Jo. Antiochenus benutzt worden ist, wird erst eine Untersuchung der vollständigen Texte zeigen. Auffällig sind die oben angedeuteten Abweichungen, auffällig ist auch der Unstand, dafs das sicher aus Jo. Antiochenus stammende Thulisorakel, welches an erster Stelle stehen müfste, in der Reihe das letzte ist.

Mit diesen Notizen würde ich mich begnügt haben, wenn ich nur die Aufmerksamkeit auf diese Orakel und Sprüche lenken wollte; indessen ich habe sie hauptsächlich deshalb erwähnt, weil ich das Thulisorakel einem andern Zwecke dienstbar machen möchte.

Als ich im Jahre 1892 das zwischen Malalas und Jo. Antiochenus bestehende Verhältnis behandelt und in folgenden Jahre einige Einsprïche, die alsbald erhoben worlen waren, zurückgewiesen hatte, glaubte ich annehmen zu dürfen, dafs die Behandlung der Frage auf ein festes Fundament gebracht sei, zumal da durch manches neue Material, wie die Wiener Troica, die gewonnenen Ergebnisse bestätigt wurden. Es störte mich nicht, als Gleye in der B. Z. V (1896) S. 452 mit den Unterschieden, die er bei einer Vergleichung des Malalastextes mit den Wiener Troica gefunden hatte, seine Hypothese zu stützen suchte, dals das Malalaswerk blofs eine Bearbeitung eines höherstehenden Werkes, nämlich der Chronik des Jo. Antiochenus, sei. Denn für jeden, der das Material kennt, war es sofort sichtbar, dals Gleye auch diesmal das Opfer seiner rätselhaften Mifsachtung des vorhandenen Materials geworden war. Eine Widerlegung in dieser Beziehung hielt ich für unnötig. Ich hatte Besseres zu thun. Aufserdem sagte ich mir, dals bei der grofsen Wichtigkeit, welche die Johannesfrage für Historiker und Philologen hat, in kürzerer Zeit doch irgend jemand die Wertlosigkeit der Gleyeschen Hypothese nachweisen würde. Aber die letzten Jahre haben mir gezeigt, dafs eine nicht mehr begreifliche Unkenntnis des Materials unter denen, welche an Jo. Antiochenus herantreten, geradezu epidemisch ist; es kann also, besonders infolge einer gebieterischen Meinungsäufseruug von einflufsreichster Seite, zu weiterer Versumpfung der un sich einfachen Frage kommen, wenn nicht energischer Einspruch erhoben wird. Deshalb habe ich mich entschlossen noch einmal zu allseitiger Abwehr auf den Plan zu treten, obgleich ich meist nur solche Dinge vorbringen kann, die schon irgendwo erwähnt oder besprochen worden sind und deshalb allgemein bekannt sein sollten. Gegen andere habe ich mich in der $\overline{\mathrm{B}}$. Z. IX S. 35̌ .u. 523, gewendet; gegen Gleye soll es hier 
geschehen durch den Nachweis, dafs Jo. Antiochenus ein Ausschreiber des Malalas ist, nichts weiter als ein gewöhnlicher und simpler Ausschreiber des Malalas.

Jo. Antiochenus hat durch die Benutzung einer ganzen Reihe von interessanten Quellen eine eigenartige, von der des Malalas grundverschiedene Weltchronik angefertigt. Unter seinen Quellen befinden sich Malalas, Petros Patrikios, Prokop, Eutrop, Ammian und ein oder mehrere Gewährsmänner für biblische und jüdische Geschichte. Seine Quellen hat er in der Weise benutzt, dafs er den Stoff bald ausführlicher wiedergab, bald auf einige Zeilen zusammenzog, bald aus anderen Quellen oder eigenem Wissen erweiterte. Sein Verfahren ist ungeheuer einfach gewesen. Trotzdem hat der Umstand, dafs Jo. Antiochenus seinen Landsmann Malalas benutzt hat, zu den kühnsten Hypothesen Anlafs gegeben. Er hat ihn aber nur für seinen Anfangsteil geplündert, weiterhin ist er anderen Hauptquellen gefolgt und hat nur noch nebenbei den Malalas herangezogen. Malalas ist also nur einer unter vielen Gewährsmännern und, wie jeder Kenner der byzantinischen Chronisten zugeben wird, unter allen gerade derjenige, dessen Verwertung am allerwenigsten auffällt. Man hat nie bestritten, dals noch im 6. Jahrhundert Johannes Ephesinus, im folgenden der Paschalchronist und Johannes von Nikiu unsern Malalas ausgeschrieben haben, warum soll nun gerade Jo. Antiochenus in einem anderen Verhältnis zu Malalas stehen?

In der That zeigt denn auch schon ein geringes Studium, dafs Jo. Antiochenus in keinem anderen Verhältnisse zu Malalas steht, als so zahlreiche andere byzantinische Chronisten. Das Ergebnis ist vollständig sicher, obgleich wir aus Jo. Antiochenus nur Exzerpte besitzen. Denn infolge der starken Benutzung, die dieser Chronist seinerseits gefunden hat, sind uns vielfach zwei, auch drei Berichte derselben Erzählung erhalten, sodafs wir für zahlreiche längere Stücke einen gesicherten Text haben. In welcher Weise für eine Vergleichung der beiden Antiochener von jeher sicherer Boden zu gewinnen war, kann man aus den Stücken ersehen, die ich in der B. Z. II S. 419 ff. besprochen habe. Auf solchem Wege gelangten wir zu der Erkenntnis, dafs die Exzerpte des Paris. 1630 bis etwa Fr. 6, 14 hauptsächlich aus Malalas stammen, fernerhin dafs die Hypothesis der Odyssee und die Wiener Troica den Text des Jo. Antiochenus bieten. Es ist nun nicht meine Absicht das ganze umfangreiche Material bis ins einzelne auszunutzen, zur Erreichung unseres $\mathrm{Z}$ weckes genügen schon wenige besonders brauchbare Stücke.

Ein Stück solcher Art ist das Thulisorakel, von dem wir hier ausgehen wollen.' Sein Hauptwert besteht darin, dafs es uns, wie 
Gelzer I S. 78 dargethan hat, über das von Jo. Antiochenus benutzte Malalasexemplar überraschenden Aufschlufs gewährt. Die Paschalchronik, die uns „den Malalastext in seiner relativ reinsten Gestalt bietet", hat den zweiten Vers des Orakels vollständig bewahrt: $\tau \alpha \tilde{v} \tau \alpha$

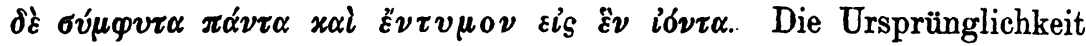
dieses Textes beweist der Cod. Paris. 1336 bei Cramer II S. 237, 18, der den Vers zwar entstellt, aber immerhin noch vollständig bietet: $\sigma v ́ \mu \varphi v \tau \alpha$

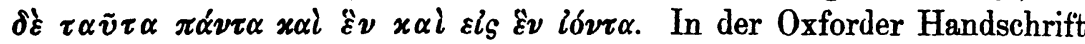

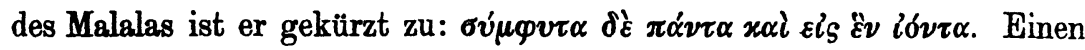
an dieser Stelle in gleicher Weise gekürzten Malalastext hat Jo. Antiochenus benutzt. Auch das Fr. 6, 9 des Paris. 1630 bietet diese Kürzung; es lälst sich aber nicht mit Bestimmtheit sagen, ob der Exzerptor diese Fassung in seinem Malalasexemplar vorgefunden hat, da er ja gleich nachher in Fr. 6, $14 \mathrm{zu}$ Jo. Antiochenus übergeht. Erwähnt sei noch, dals der Paris. 1336 (S. 237, 12) die Erzählung gekürzt hat und mit

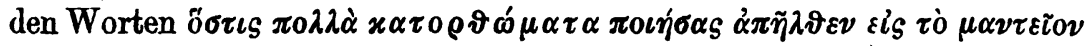

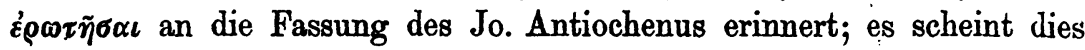
aber Zufall zu sein. Hier bewies die Beschaffenheit des Malalasexemplars die Abhängigkeit des Jo. Antiochenus von Malalas; dafs auch abweichende Lesarten zu diesem Ergebnis führen, habe ich in der B. Z. IX S. 368/9 an einer Variante in dem Heraklesstücke gezeigt.

In dem Thulisorakel des Jo. Antiochenus finden sich ferner zwei Angaben, die bei Malalas fehlen. Wir erfahren, dafs dieses Orakel das des Serapis gewesen sei und dafs die Insel Thule ihren Namen dem König Thulis verdanke. Wie haben wir über dieses Plus zu urteilen? Schon die textgeschichtlichen Verhältnisse lassen keinen Zweifel, dals wir es hier mit späteren Zusätzen zu thun haben. Denn da sich diese Angaben weder in dem besseren Texte der Paschalchronik, noch in dem schlechteren des Oxoniensis, der die Fassung des von Jo. Antiochenus benutzten Exemplars zeigt, vorfinden, so können sie nur Zusätze eines Schriftstellers sein, der die Überlieferung seiner Hauptquelle aus eigenem Wissen oder anderen Quellen erweitert hat. Aber auch andere Umstände beweisen dies. Die Bezeichnung der Orakelstätte verrät sich als spätere Zuthat auch deshalb, weil bei Mal. S. 65 bei Erwähnung eines aulcren Orakels (No V) der Name der Gottheit gleichfalls fehlt. Aufserdem sieht man ohne weiteres, dafs es sich um Orakelstätten handelt, deren Vorbild die Orakelstätte des delphischen Apollo gewesen ist. Denn auf S. 65 wird in Memphis die Pythia befragt und auf S. 25, wo es sich offenbar nicht einmal um eine ägyptische Orakelstätte

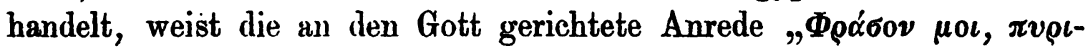

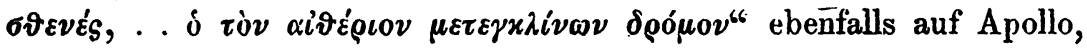


jedenfalls nicht auf Serapis. Von diesem Gotte, dessen Dienst sich erst seit der Zeit der Ptolemäer entwickelt und verbreitet hat, hat auch die Urquelle, aus welcher die Götterliste bei Malalas stammt, nichts gewulst. Denn Malalas zitiert S. 25, 11 Manetho, und Gelzers Erörterungen I S. 193/4 zeigen, dafs wirklich bei Malalas die echte Götterliste Manethos vorliegt, wenn auch lückenhaft.

Ein späterer Zusatz ist auch die nur aus Suidas bekannte Angabe ïber dir Insel Thule, nur lärst sich ihre Einfügung durch Jo. Antiochenus nicht so bestimmt behaupten, weil dieser Zusatz in dem von Bentley veröffentlichten Orakel fehlt. Er ist aber zweifellos johanneisch. Denn Zusätze geographischen Inhalts liebt Jo. Antiochenus, wie viele Byzantiner. So enthält Fr. 6 No 15 Paris. 1630 die Erklärung $\varepsilon^{\xi \xi}$ oz

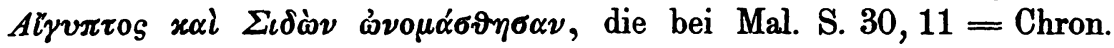
Pasch. S. 76, 18 fehlt; in den Wiener Troica S. 6, 26 heifst es von

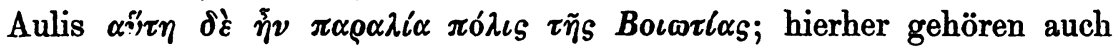
die ethnographischen Erklärungen in Fr. 11 No 3 Paris. 1630 हैं

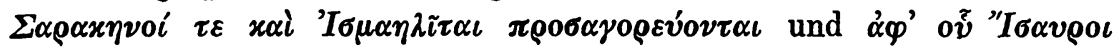

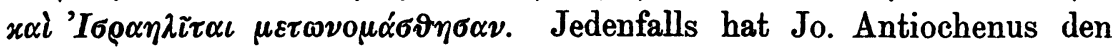
Stoff seiner Hauptquelle durch Zusätze gern erweitert. Iç will dies noch an zwei ganz verschiedenartigen Beispielen nachweisen, von denen das erste zum Teil auch geographischen Charakter trägt.

1) Eine charakteristische Eigentümlichkeit, welche das Werk des Jo. Antiochenus von dem des Malalas schon in dem Anfangsteile, worin ja Malalas fast allein zu Worte kommt, aufs schärfste trennt und uns jede Möglichkeit abschneidet die Chroniken der beiden Antiochener zu identifizieren oder in dem Malalaswerke eine Vulgärbearbeitung der Chronik des Jo. Antiöchenus zu sehen, ist die eigenartige Behandlung der biblischen und jüdischen Geschichte. Sie tritt aufs deutlichste hervor 1) in dem von Abraham und Lot handelnden Mittelstücke des Fr. 9 Virt.; 2) in dem über Sodom und Gomorrha handelnden Schlusse des Fr. 9 Virt., womit Fr. 11, 2 Paris. 1630 stimmt; 3) in dem über Simson und Delila handelnden Fr. 16 Virt., womit das Fr. 15, 5 Paris. 1630 stimmt; 4) in dem die Könige Saul, David, Salomo behandelnden Fr. 18 Virt., dessen über David handelnder Abschnitt auch im Anfang des Titels De Insidiis erscheint und dessen über Salomo handelndes Stück mit Fr. 17 Paris. 1630 stimmt (B. Z. II S. 416/8). Man beachte, dafs es lauter konstantin ische Fragmente sind, mit denen hier der Paris. 1630 stimmt, und dafs alle Suidasglossen johanneischen Ursprungs aus koustantinischen Titeln stammen (vgl. B. Z. VI S. 352/3).

Einen solchen biblischen Abschnitt nun hat Jo. Antiochenus aus Prokop erweitert. Es handelt sich hier um das wichtige Stück, das ich 
in der B. Z. II S. 422 aus der Suidasglosse X $\alpha \nu \alpha \alpha^{\prime} \nu$, dem Fr. 11, 5 Paris. 1630) und dem Fr. 12 Salm. als johanneisch erwiesen habe. Jo. Antiochenus erzählt da, wie nach Mosis Tode von Josua die Israeliten in das gelobte Land geführt und die vertriebenen Kananäer nach Afrika gedrängt worden seien, und berichtet dann, dafs noch jetzt in Numidien

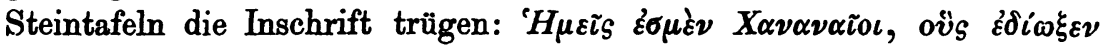

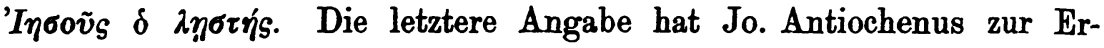
weiterung seiner Bibelerzählung aus Prokops Bell. Vandal. II cp. 10 genommen. Dafs Prokop und kein anderer Schriftsteller der Gewährsmann des Antiocheners gewesen ist, ergiebt sich daraus, dafs Euagrios in seiner Historia Ecclesiastica IV 18 Prokops Angabe mit der ausdrücklichen Bemerkung zitiert, vor Prokop habe niemand solches über-

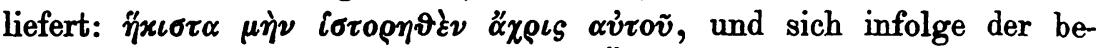
stimmten Angaben Prokops über die Örtlichkeit, den Schriftcharakter und Wortlaut der Inschrift eingeredet hat, Prokop behaupte die Inschrift

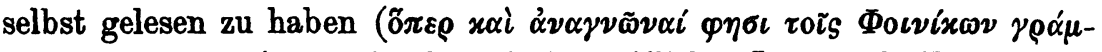

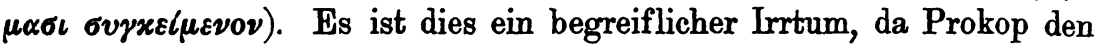
Feldherrn Belisar auf seinem Kriegszuge gegen die Vandalen als Geheimschreiber begleitet hat und so bestimmte Angaben macht, als hätte er die Örtlichkeit selbst gesehen und die Inschrift selbst gelesen. Auf Prokop gehen auch, mittelbar oder unmittelbar, alle Angaben zurück, die wir bei Byzantinern über diese Inschrift finden. Euagrios zitiert ihn; Jo. Antiochenus hat ihm diese Angabe entnommen, weil er Prokops Bell. Vandal. auch sonst benutzt hat (Fr. 196 Salm. u. Fr. 200 Salm.). Aus Prokop hat ferner Theophanes S. 200, 16 geschöpft, jedoch

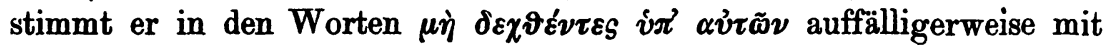
I A $\mu \eta े\rangle$

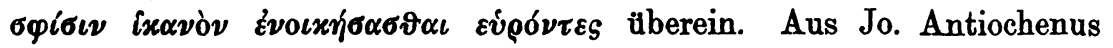
ist diese Angabe durch Vermittelung der konstantinischen Exzerpte zu Suidas gelangt. Die Chronik des Paris. 1712 bringt die Angabe an zwei Stellen: auf Fol. 37 nach Euagrios und auf Fol. 135/6 nach Theophanes. Von fremden Autoren hat Moses von Khoren die Inschrift in seiner Geschichte Armeniens erwähnt. Carrière hat in seiner Schrift Nourelles sources de Moïse de Khoren, Supplément, 1894 S. 30/40 die Quellenfrage sehr eingehend behandelt, aber ohne die Ergebnisse der auf Jo. Antiochenus gerichteten Forschung (bes. B. Z. II S. 422) zu kennen. Ich zweifle nicht, dafs die Notiz auf Jo. Antiochenus zurückgeht und von Moses mit neuen Zügen (vgl. Carrière S. 37) ausgestattet worden ist.

Die litterargeschichtliche Mitteilung des Euagrios ist für uns höchst wertvoll, denn sie bestätigt gläuzend die von Köcher, Sotirindis und 
Boissevain ausgesprochene und von mir geteilte Ansicht, dafs Prokop ein Gewährsmann des salmasischen Jo. Antiochenus gewesen ist. Leider habe ich sie erst nach der Veröffentlichung meines Aufsatzes "Jo. Antiochenus Fr. 200 Salm. und Prokop“ B. Z. II S. 591 kennen gelernt. Man wird es begreiflich finden, dafs durch diese litterargeschichtliche Notiz des Euagrios meine Überzeugung, dafs nicht die konstantinischen Exzerpte, sondern die salmasischen Exzerpte die richtige, echte Fortsetzung des gemeinsamen Anfangsteiles darstellen, bis zur Unerschütterlichkeit gefestigt worden ist, zumal da Gleyes Versuch das fatale Zeugnis des Euagrios zu entwerten und eine gemeinsame ältere Quelle in nebelhafter Ferne zu zeigen (S. 462/3) vollständig milsglückt ist. Da soll vor

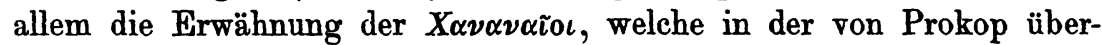

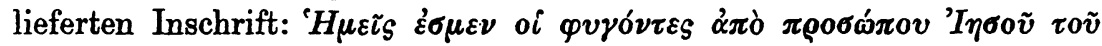

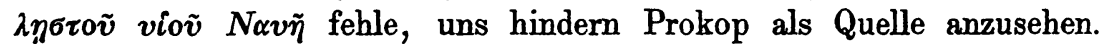
Als ob nicht die Bibel (Josua cp. 10/12) von der Eroberung Kanaans und der Vertreibung der Kananäer genügend erzählte! Da soll ferner bei Kedren S. 93, 15/23 sogar noch ein der Quelle des Prokop verwandter Bericht vorliegen. Eine merkwürdige Kenntnis des Materials! Kedrens Bericht geht auf das Buch Josua cp. 10/12 zurück, aber auch nicht unmittelbar; er ist, wie schon Gelzer II S. 364 in seiner Quellenanalyse Kedreus angegeben hat, aus Georg Monachos S. 101, 12/20 genommen. Und nun beantworte man sich zuletzt noch folgende Fragen. Euagrios hat nicht blols Prokop, sondern auch den nach Gleyes Ansicht mit Jo. Antiochenus identischen Jo. Rhetor benutzt; in beiden Quellen hat ex

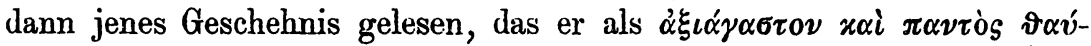

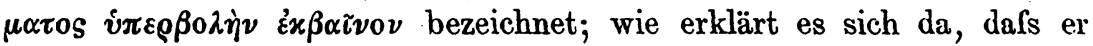
den Prokop als älteste Quelle anführt und nicht vielmehr den von ihm so häufig zitierten Jo. Rhetor? Und weiter frage ich: Der Antiochener Euagrios ist ein belesener und unterrichteter Kirchenhistoriker, er ist ferner der Freund und Berater der antiochenischen Patriarchen Gregor und Anastasios (559-599), die sich beide einen Namen in der Litteratur gemacht haben; in den höchsten litterarischen und theologischen Kreisen von Antiochien ist man also in Verblüffung über die Angabe Prokops - und da soll wenige Jahrzehnte vorher ein antiochenisches Mönchlein in einer Chronik, die noch dazu von Euagrios ausgeschrieben und zitiert wurde, diese Angabe nach einer älteren Quelle gemacht haben? Mau verschone uns doch endlich mit Hypothesen, die weder gehauen noch gestochen sind, und nehme die Sache so einfach, wie sie liegt: Der Jo. Rhetor des Euagrios ist unser Jo. Malalas, der die ersten 17 Bücher seines Werkes vor 533 garschrieben hat; Jo. Antiochenus aber hat 80 Jahre nach diescr Zuit 
gelebt und aufser Malalas und anderen Autoren auch Prokop als Quelle benutzt.

2) In der Erzähhlung der troischen Sagen, die Jo. Antiochenus hauptsächlich nach Malalas gegeben hat, ist Homer, der am Schlusse des Fr. 23 Virt. ausdrücklich als Quelle zitiert wird, zur Erweiterung herangezogen worden. Schon in der B. Z. II S. 426 habe ich dargelegt, dafs Jo. Antiochenus den Tod des Patroklos nach Homer erzählt haben müsse; die Wiener Troica sind gerade in diesem Abschnitte lückenhaft, sie haben aber doch durch die Erwähnung der Leichenfeier des Patroklos eine Bestätigung meiner Vermutung gebracht (B. Z. IV S. 26). Damit ist aber die Benutzung Homers noch nicht erschöpft. Auf Il. XXIV $324 \mathrm{ff}$. weist in den Wiener Troica S. 7, 27 auch die Angabe, dafs Priamos auf einem vierrädrigen Wagen nächtlicher Weile zum Zelte des Achilles aufgebrochen sei. Diese Angabe ist von Jo. Antiochenus in den Anfang der ganz anders gearteten Erzählung des Malalas aufgenommen worden. Von dieser Erweiterung kann sich jeder leicht überzeugen, da uns bei Dictys-Septimius III $20 \mathrm{ff}$. die Quellenerzählung des Malalas zur Vergleichung vorliegt. Darauf werden wir sogleich in dem wichtigsten Teile unserer Beweisführung zurückkommen.

Aufser Homer hat Jo. Antiochenus zur Ergänzung der aus Malalas genommenen Troica auch noch andere Autoren herangezogen, nämlich Dictys Cretensis, Vergil und eine christliche Quelle, die ihm das Hilfegesuch des Priamos bei David geliefert hat. Auf die Verwertung dieser Quellen gehe ich hier nicht weiter ein, weil die Betrachtung eines kleinen Abschnittes der Wiener Troica die Abhängigkeit des Jo. Antiochenus von Malalas so schlagend erweisen wird, dafs ich jede andere Auseinandersetzung für überflüssig halte. Nur das eine will ich bemerken, dals zu einem Zweifel an der "subsidiären“ Benutzung des Dictys, wie ihn Gleye S. 454/5 ausgesprochen hat, auch nicht der geringste Anlals vorliegt. Nichts ist bei den Byzantinern häufiger als eine mittelbare und zugleich unmittelbare Benutzung derselben Quelle, von Malalas angefangen bis Zonaras. Auch erregt die Quelle selbst keinerlei Bedenken. Denn dafs Dictys bekannt war, zeigt die lateinische Bearbeitung des Septimius, und bei Jo. Antiochenus kann die Verwertung des Dictys um so weniger auffallen, als die Dictyserzählung 80 Jahre vorher in derselben Stadt ganz unbestreitbar von Malalas verwertet worden ist.

Es ist von jeher betont worden, dafs Malalas stellenweis mit Dictys-Septimius überraschend stimmt, auch in solchen Abschnitten, die er unmittelbar aus Sisyphos von Kos geschöpft hat. In der B. Z. I S. 413 habe ich diese Frage behandelt und namentlich auf Dictys III 
15-IV 11 verwiesen. Man hätte nun glauben sollen, dafs niemand die Wiener Troica zum Gegenstande einer Quellenuntersuchung machen werde, ohne die lateinische Ephemeris zur Seite zu haben. Aber wenn es sich um Jo. Antiochenus handelt, wird das Unglaublichste, das Unbegreiflichste fertig gebracht. So hat Gleye S. 456/8 einen Abschnitt der Wiener Troica mit dem Texte des Malalas verglichen, ohne eine Ahnung zu haben, dafs bei Dictys-Septimius die Quellenerzählung zur Kontrolle vorliegt. Seine Ausführungen sind deshalb wertlos. Eine Vergleichung der Erzählungen des Malalas und Jo. Antic.chenus mit der ursprünglichen Quellenerzählung führt zu ganz entgegengesetzten Ergebnissen. Ich erwähne nur das wichtigste.

Nachdem Jo. Antiochenus S. 7, 25 die Bestattung des Patroklos nach Homer erwähnt hat, fügt er S. 7, 27-S. 8, 2 den Besuch des Priamos bei Achill in der Weise an, dafs er die oben bezeichneten Homerangaben in den Anfang der Malalaserzählung einschiebt. Die letztere hat er dabei vereinfacht und gekürzt. Während z. B. bei Dictys III 20 und Mal.S. 123/4 Priamos sich mit Polyxena, Andromache und deren beiden Söhnen Astyanax und Laodamas am hellen Tage (lucis principio,

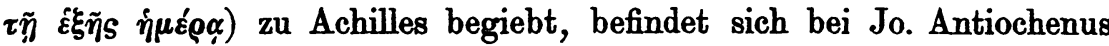
nur Polyxena in der Begleitung des Priamos. In den Zeitangaben bemerken wir sogar einen Widerspruch, der die flüchtige Arbeitsweise des Antiocheners verrät. An der Spitze steht nämlich die Zeitangabe

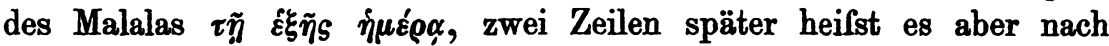

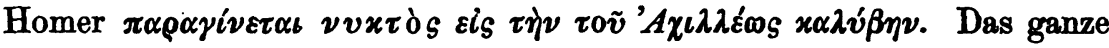
Stück ist für das Verfahren des Jo. Antiochenus charakteristisch. Er vereinfacht und kürzt die ausführliche Erzählung seiner Hauptquelle, erweitert aber diese zugleich durch Angaben aus einer Nebenquelle und schafft auf diese Weise eine neue Erzählung. Aber sein eigenmächtiges Verfahren erstreckt sich nicht blols auf die Gestaltung des ganzen Stoffes, auch Einzelheiten werden von ihm in selbständiger Weise geändert oder infolge flüchtiger Arbeit entstellt. Über das Bittflehen Polyxenas berichten unsere drei Autoren so:

Dictys III 24

Polyxena ingresso

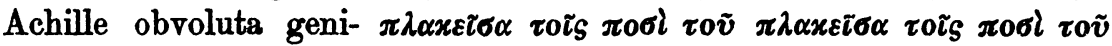

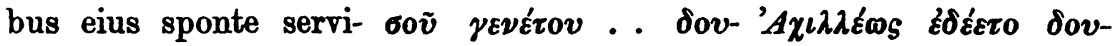

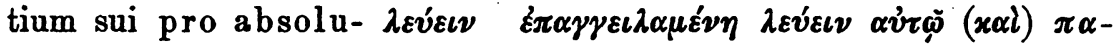

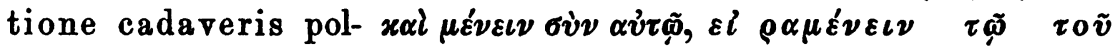
licetur.

$$
\text { Mal. 124, } 13
$$

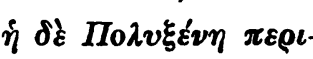

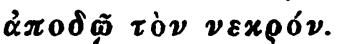

Jo. Ant.

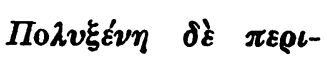

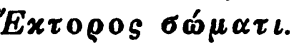

Malalas stimmt hier mit Dictys, Jo. Antiochenus hat also seine VorByzant. Zeitschrift X 1 a. 2. 
lage entstellt. - Ähnlich wie in dem eben besprochenen Stücke ist Jo. Antiochenus auch in den 'folgenden Stücken verfahren.

An die Rückkehr áes Priamos und die Bestattung Hektors schliefst sich S. 8, 2 die Erzählung von der Amazonenkönigin Penthesilea. Dictys IV 2 und Mal. S. 125, 18 beginnen damit einen neuen Abschnitt und stimmen in der Erzählung selbst aufs schönste überein; Jo. Antiochenus bringt dagegen wichtige Änderungen an. Erstens verknüpft er die Erzählung mit der vorhergehenden aufs engste so, dals Priamos Subjekt

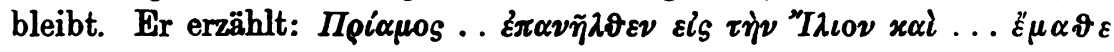

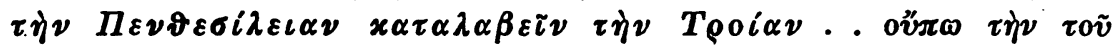

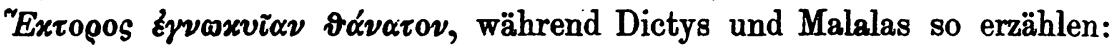

Interim per eosdem dies Penthesilea .. supervenit. quae postquam interemptum Hectorem cognovit, .. regredi domum cupiens etc.

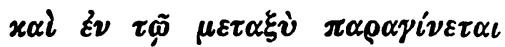
$\dot{\eta} \Pi \varepsilon \nu \vartheta \varepsilon \sigma i \lambda \varepsilon \iota \alpha . \mu \mu \alpha \vartheta 0 \tilde{v} \sigma \alpha \quad \delta \xi$

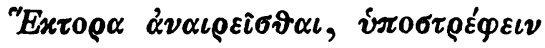

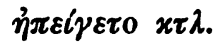

Zweitens schiebt Jo. Antiochenus den Tod Polydors mitten in die Penthesilea-Episode ein, was ihn selbstrerständlich zu eigenmächtigen Änderungen veranlafst, während Dictys II 18/27 und Mal. S. 103, 7 (Ekloge S. 204, 7) übereinstimmend den Tod Polydors an die Rückkehr des Ajax von seinen Beutezügen anschliefsen. Wie Jo. Antiochenus dazu gekommen ist den Tod Polydors an einer ganz unpassenden Stelle in eine Erzählung einzufügen, die bei Dictys und Malalas ganz geschlossen verläuft, kann ich nicht erklären; vielleicht ist es mit eine Folge davon, dafs sich Jo. Antiochenus auf S. 7, 15, wo sich der Tod Polydors hätte anschliefsen müssen, anderen Quellen zugewendet hat.

Weiterhin folgt S. 8, 14 die Memnon-Episode. Ihr Schlufs ist hochinteressant. Bei Dictys IV 6-9 und Mal. S. 129 ist der Gang der Ereignisse folgender: Memnon und seine Mannen werden in einer Schlacht vernichtet, die Trojaner erbitten die Auslieferung der Toten, diese werden verbrannt; nach einigen Tagen (post paucos dies, ódíwov $\dot{\eta} \mu \varepsilon \rho \tilde{\nu} \nu \delta \iota \alpha \delta \rho \alpha \mu 0 v \sigma \tilde{\omega} \nu$ ) wird eine neue Schlacht geschlagen, viele gehen im Skamanderflusse zu Grunde, Troilos und Lykaon werden getötet. Dictys und Malalas berichten also übereinstimmend von zwei Schlachten, Jo. Antiochenus zieht dagegen die beiden Schlachten in eine einzige

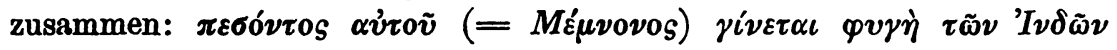

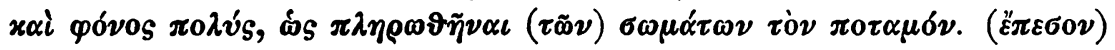

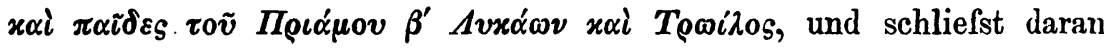
die bei Dictys und Malalas zwischen den beiden Schlachten stehende Bitte der Trojaner um Waffenstillstand zur Bestattung der Toten. 
Es folgt S. 8, 27 der Tod Achills in Heiligtume des thymbräischen Apoll. Aufser an zwei Stellen, die ich zitieren werde, stimmen Septimius IV 10/11 und Malalas S. 130/1 überein. Jedoch sind bei letzterem mehrere Einzelheiten zu finden, die sicherlich auch in dem griechischen Dictys des Septimius gestanden haben. So finden die Worte in IV 10 "tempus nanctus" nur durch folgende Angabe des Malalas

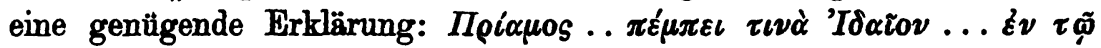

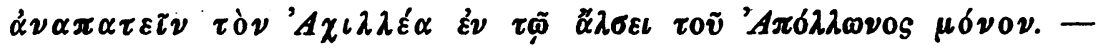
Achill verhandelt im Heiligtume Apollos mit Idäos über Polyxena. Als die Griechen dies bemerken, erfalst sie Unruhe; es regt sich sogar der Verdacht, Achill könne Verrat üben. Sie entsenden den Ajax, Diomedes und Odysseus,

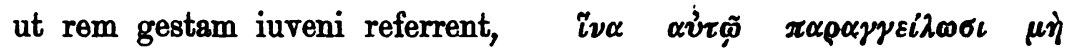

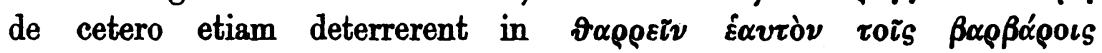
colloquio clam cum hostibus agere. $\mu \delta ́ v 0 \nu$.

Die Gesandten erwarten Achill vor dem Heiligtume (ante templum,

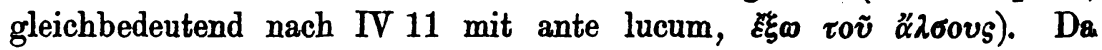

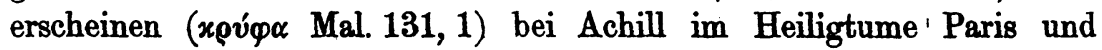
Deiphobos, scheinbar um die mit Idäos getroffenen Vereinbarungen zu bestätigen. Achill fürchtet nichts Böses (in sacro Apollinis nihil hostile

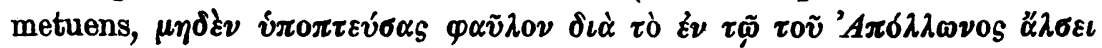
Eival). Aber am Altar wird er von Deiphobos umarmt und von Paris getötet. Die Mörder eilen auf einer anderen Seite, als wo sie gekommen

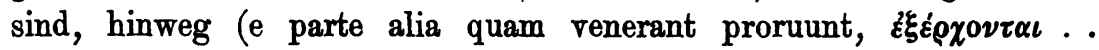

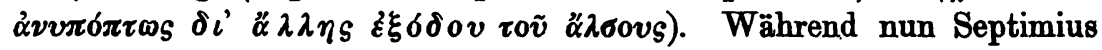
nur sagt: in civitatem recurrunt, berichtet Malalas ausführlicher: $\dot{\omega} s$

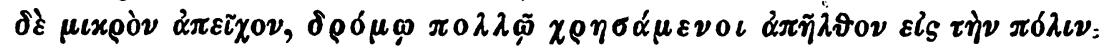
Odysseus bemerkt die beiden und ruft dem Ajax und Diomedes zu:

„Non temere est, quod hi turbati ac trepidi repente prosiluere".

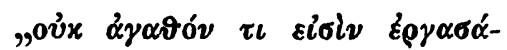

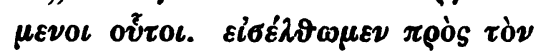

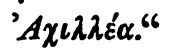

Die Helden eilen in das Heiligtum und finden Achill in seinem Blute liegen:

Nun vergleiche man diese an Einzelheiten reiche und klare Erzählung mit der gekürzten und entstellten Erzählung des Jo. Antiochenus S. 8, 31. Die drei Fürsten warten nicht vor dem Haine auf Achill, sondern begeben sich hinein und unterhandeln mit Achill:

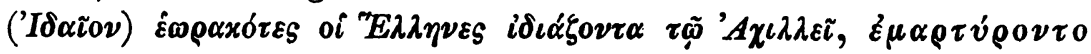

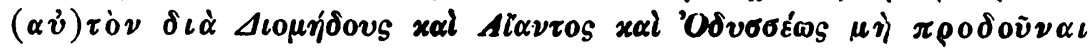




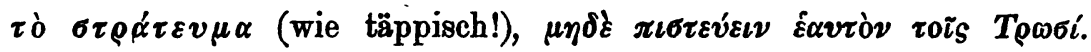

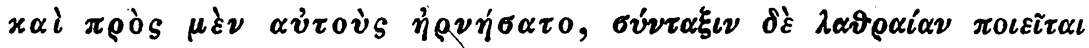

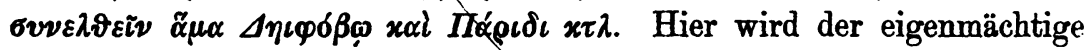
Ausschreiber zum selbständigen Errfinder. In welcher Weise die Fürsten die Verhandlungen mit Idäos gestồ haben und weshalb sie wieder weggegangen sind, erfahren wir nicht; jedenfalls finden wir sie gleich nachher aufserhalb des Heiligtums, da die Ermordung Achills sich so vollzieht wie bei Malalas. Als Odysseus die fittchtenden Mörder sieht,

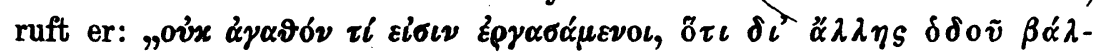

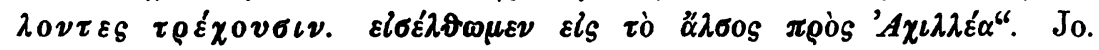
Antiochenus bietet die Worte des Malalas, hat aber ein Sätzchen eingeschoben. Dieses hat er aber nicht etwa aus der Quelle äberkommen, sondern er hat es aus der vorausgehenden Erzählung des Malalas, die er gekürzt hatte, eingefügt, um den Schreckensruf des Odysseus: „Die haben nichts Gutes verrichtet. Hinein!“ zu begründen. Dabei hat er aber übersehen, dals die eingefügten Worte im Munde des Odysseus widersinnig sind, da die Ankunft der beiden Trojaner unbemerkt erfolgt ist."

So viel zur Beleuchtung dessen, was Gleye S. 456/8 vorgetragen hat. Ich könnte vieles auch gegen Gleyes übrige Beweise (S. 453/6) vorbringen*), z. B. gegen seine Billigung der komischen Angabe, welche Pelops und Europa zu Stammeltern eines Heroengeschlechts macht (S. 453); indessen wozu eine überflüssige Widerlegung ganz unzulänglicher Argumente! Die Vergleichung jenes kleinen Abschnittes der

*) Nachtrag: Einige Monate nach dem Abschlusse meines Aufsatzes ist in dem Jahresbericht des Andreas-Realgymnasiums zu Berlin, Ostern 1900, unter dem Titel: „Neue Untersuchungen zur Dictys- und Daresfrage. - I. Dictys Cretensis bei den Byzantinern" eine Abhandlung von W. Greif erschienen, die für unsern Gegenstand von Wichtigkeit ist. In der Beurteilung des zwischen Malalas und Jo. Antiochenus bestehenden Verhältnisses ist Greif an moine Seite getreten. Hr wendet sich deshalb S. 25/7 ebenfalls gegen Gleye, widerlegt aber im ganzen nur solche Argumente, die ich mit Absicht gerade unbeachtet gelassen habe. Denn auch ihm, dem Dictysforscher, ist es entgangen, dafs bei Dictys-Septimius die Quellenerzählung des Malalas zur Kontrolle vorliegt. Unsere Arbeiten ergänzen sich deshalb in willkommener Weise. - Auch für die Frage, welche Quellen Jo. Antiochenus in seinen Troica nebenbei verwendet hat, liefert Greif einen Beitrag. Er glaubt S. 27/8 in der Angabe des Jo. Antiochenus S. 9, 6, dafs Pyrrhos den Philoktet aus Lemnos mitgebracht habe, den Finflufs des älteren Philostrat zu erkennen. Vielleicht handelt es sich aber nur 'um eine Erfindung des Antiocheners. Der historische Gang der Ereignisse erforderte die Ankunft des Pyrrhos (Dictys IV 15), und da Jo. Antiochenus für die aus Malalas S. 110 (Dictys IV 19) angeschlossene Erzählung Philoktet brauchte, so war es für ihn dus einfachste Pyrrhos und Philoktet in Gesellschaft reisen zu lassen. 
Wiener Troica mit der Erzählung des Malalas und des Dictys-Septimius zeigt zur Genüge nicht nur die Abhängigkeit des Jo. Antiochenus von Malalas, sondern auch seine Eigenart und Selbständigkeit in der Verwertung der benutzten Quellen. Wer sich die geringe Mühe macht diese Vergleichung nachzuprüfen, mufs zu der Erkenntnis kommen, dafs die Behauptung, das Malalaswerk sei eine Vulgärausgabe der höherstehenden Chronik des Jo. Antiochenus, nicht eine Hypothese ist, die bei einem umsichtigen Studium des Materials langsam emporgereift ist, sondern ein Phantasiegebilde, das auf einem Nebelritte durch Wolkenkuckucksheim geschaut worden ist.

Leipzig.

Edwin Patzig.

Anmerkung der Redaktion: Wenn ich nicht irre, bedarf die vielgeplagte Malalas-Johannesfrage dringend der Kuhe. Die Polemik yermag keine wesentlichen neuen Momente mehr ins Feld zu führen und droht durch die persönliche Verstinmung der Streitenden immer unfruchtbarer zu werden. Damit, dafs alte Argumente öfter wiederholt werden, gewinnen sie nicht an Beweiskraft. So lange nicht durch handschriftliche Funde ein wirklich neues Material beigeschafft wird, ist $\mathrm{m}$. E. keine Verständigung $\mathrm{zu}$ erzielen. Ich sehe mich daher zu dem ungewöhnlichen Schritte genötigt, die Diskussion über diese Crux der byzantinischen Quellenforschung in der B. Z. für geschlossen $z$ u erklären, bis der angedeutete günstige Fall cintritt. 